\title{
A Study on Efficacy of Phenytoin on Chronic Non Healing Diabetic Ulcer
}

\author{
Dr. Balasubrahmanya. K.S., Dr. Mano Ananth A. B, Dr. Parveen Pawar, \\ From Mysore Medical College And Research Institute
}

\begin{abstract}
Abstarct:
Background: Chronic non healing ulcer is one the commonly faced surgical conditions by surgeons. It is one of the major cause for morbidity in diabetic patients. Inspite of various types of dressing agents available, choosing an appropriate dressing is a challenging aspect in the management of diabetic ulcer. Topical phenytoin causes rapid wound remodeling in non healing ulcers and its application in wound healing needs further study.

Objective : To assess the efficacy of topical phenytoin compared to conventional wound care in improving the healing process and to prove it as a relatively low cost and easy to use option in the management of diabetic ulcers.

Methodology: Our study is a randomized prospective study was conducted on 100 patients with non healing chronic diabetic ulcer. The patients are randomly divided into two groups study and control group ( Study phenytoin; Control - $5 \%$ povidone iodine). Wound measurement and culture growth was taken on day one and end of 14 th day. Mean reduction in ulcer area and culture growth at the end of 14 days was noted.

Results : There was no statistical difference in the baseline characteristics like age, sex, initial and final wound area of the ulcer between the two groups. The mean reduction in wound area was $6976.1 \pm 1441.5 \mathrm{~mm}$ in patients treated with topical phenytoin dressings and $1960.7 \pm 280.4 \mathrm{~mm}$ in patients treated with $5 \%$ povidone iodine dressings, which is statistically significant $(p<0.001)$.

Conclusions: Phenytoin has a fibroblast proliferating and anti bacterial property which can be used in faster healing for chronic diabetic ulcers as topical agent thereby reducing its systemic side effects.
\end{abstract}

Keyword: Diabetic ulcers, povidone iodine, topical phenytoin, Wound $\backslash$ area

\section{Introduction}

Chronic non healing ulcers are one of the common surgical conditions encountered by surgeons which is more commonly occurs in diabetic patients and nearly one fifth of diabetic patients admitted in hospitals are suffering from non healing foot ulcers and leading to lower limb amputations in many cases (1). Various methods has been proposed by many authors over a period of time for healing and prevention of diabetic ulcer.Since diabetes is a systemic disease which causes neurological, vascular and immunological complications favouring the foot ulcers to get arrested in inflammatory stage of healing. Nearly $15 \%$ of all diabetic develop ulcers in their life time and the risk of lower limb amputations is 15 fold increases when compared to non diabetics( 2). The management of wound and wound dressing is an important aspect of diabetic ulcer management, which is neglected many a time. Care of the wound involves management of the ulcer, care of the exudates and knowledge and rational use of myriad dressing materials.

During the last two decades a wide variety of innovative dressings have been introduced. Diabetic ulcers are the indication for $50 \%$ of non traumatic amputations. There is a need for evaluation of new method for treating these ulcers which are economical and more effective in increasing healing rate and decreasing the amputation rate. Some studies on topical phenytoin have shown increased healing rate in chronic foot ulcers than other conventional dressings $(3,4,5,6,7,8,9)$.Few studies stated that topical phenytoin increased the healing rate of diabetic foot ulcers, but there was no difference in complete healing of the ulcers compared to other conventional dressings. $(4,5)$

Phenytoin acts by stimulating fibroblasts enhancing granulation tissue formation, decreasing collagenase activity (6) Systemic absorption of phenytoin on topical use in diabetic ulcer was not significant. Other side effects noticed on use of topical phenytoin in diabetic ulcer were transient burning sensation initially and hypergranulation. $(5,6,7,8)$. Though many studies are conducted on using topical phenytoin in chronic leg ulcers only few studies are conducted on diabetic ulcers .

\section{Objective Of The Study}

To assess the efficacy of topical phenytoin compared to conventional wound care in improving the healing process and to prove it as a relatively low cost and easy to use option in the management of diabetic ulcers. 


\section{Methodology}

Our is a prospective randomized comparative study included 100 patients with diabetic ulcers satisfying all the inclusion criteria mentioned below after obtaining consent and clearance from the ethical committee.

\section{Inclusion criteria :}

Patients with chronic ulcers (ulcers of 4 weeks duration) with diabetes mellitus with wound size $<5 \%$ of total body surface area.

\section{Exclusion criteria :}

- Chronic non-healing wounds of other etiology

- Diabetes mellitus with gangrenous changes.

- Wounds with osteomyelitis.

- Wounds with poor vascularity

- Other co-morbid conditions like renal failure, generalized debility and other factors, which adversely affect wound healing.

The data was collected from 100 patients who are having diabetic ulcers satisfying the inclusion criteria mentioned above. The whole sample population was divided into group A and group B randomly. Group A contain 50 patients and Group - B contain 50 patients. All patients underwent detailed clinical examination and relevant investigations and the wounds were thoroughly debrided and the ulcer dimensions as well as the surface area assessed using measuring tape, before both types of dressings were applied. The control group and study group were subjected to daily dressing. Discharge is sent for culture and sensitivity. Empirical antibiotics are started with ciprofloxacin and metronidazole changed to sensitive antibiotics after sensitivity report. The patients were followed up for 2 weeks in both study and control groups.

\section{Application of Dressing:}

Group A is dressed with topical phenytoin (study group) and group B with povidone iodine (control group)

\section{Topical Phenytoin}

Phenytoin sodium tablet was crushed and dissolved in $5 \mathrm{ml}$ of normal saline to form a suspension. Sterile gauze was soaked in the suspension and spread evenly over the ulcer and left for 24 hours till the next dressing Dosage of phenytoin depend on the surface area of ulcer

- 0 to $5 \mathrm{~cm}^{2}-100 \mathrm{mg}$

- 5.1 to $9 \mathrm{~cm}^{2}-150 \mathrm{mg}$

- 9.1 to $15 \mathrm{~cm}^{2}-200 \mathrm{mg}$

- $>15 \mathrm{~cm}^{2}-300 \mathrm{mg}$.

Control group Dressing was done with 5\% povidone iodine ( betadine ) once a day. Before applying both dressing daily wound is cleaned with normal saline and debridement is done if necessary. Ulcer size is measured initially and at the end of 14 days, size is recorded. Size is measured twice and mean of two is taken. Wound is also observed for granulation tissue, discharge at the end of 14 days are recorded, wound discharge is sent for culture and sensitivity on $14^{\text {th }}$ day of treatment.

\section{Results}

A total of 100 patients satisfied the selection criteria were involved in the study, analysis was done by using Cramer's V test, Independent-Samples T Test, Repeated measure Anova. There was no statistical difference in the baseline characteristics like age, sex , initial and final wound area of the ulcer between the two groups

\begin{tabular}{|l|l|l|l|l|l|}
\hline \multicolumn{2}{|l|}{ Table 1: Culture growth on day 1 } & Total \\
\hline \multicolumn{2}{|l|}{} & DRESSING & \\
\cline { 3 - 6 } \multicolumn{2}{|l|}{ PHENYTOIN } & $\begin{array}{l}\text { POVIDON } \\
\text { E IODINE }\end{array}$ & \\
\hline Day 1_CUL & $+\mathrm{V}$ & Count & 46 & 45 & 91 \\
\cline { 3 - 6 } & $\mathrm{e}$ & $\%$ within DRESSING & $92.0 \%$ & $90.0 \%$ & $91.0 \%$ \\
\hline
\end{tabular}


A Study On Efficacy Of Phonation On Chronic Non Healing Diabetic Ulcer

\begin{tabular}{|l|l|l|l|l|l|}
\hline & \multirow{2}{*}{-Ve } & Count & 4 & 5 & 9 \\
\cline { 3 - 6 } & & $\%$ within DRESSING & $8.0 \%$ & $10.0 \%$ & $9.0 \%$ \\
\hline \multirow{3}{*}{ Total } & Count & 50 & 50 & 100 \\
\cline { 3 - 6 } & \% within DRESSING & $100.0 \%$ & $100.0 \%$ & $\begin{array}{l}100.0 \\
\%\end{array}$ \\
\hline
\end{tabular}

CRAMER'S V VALUE -0.035 P -0.727

GRAPH 1 - CULTURE ON DAY ONE :

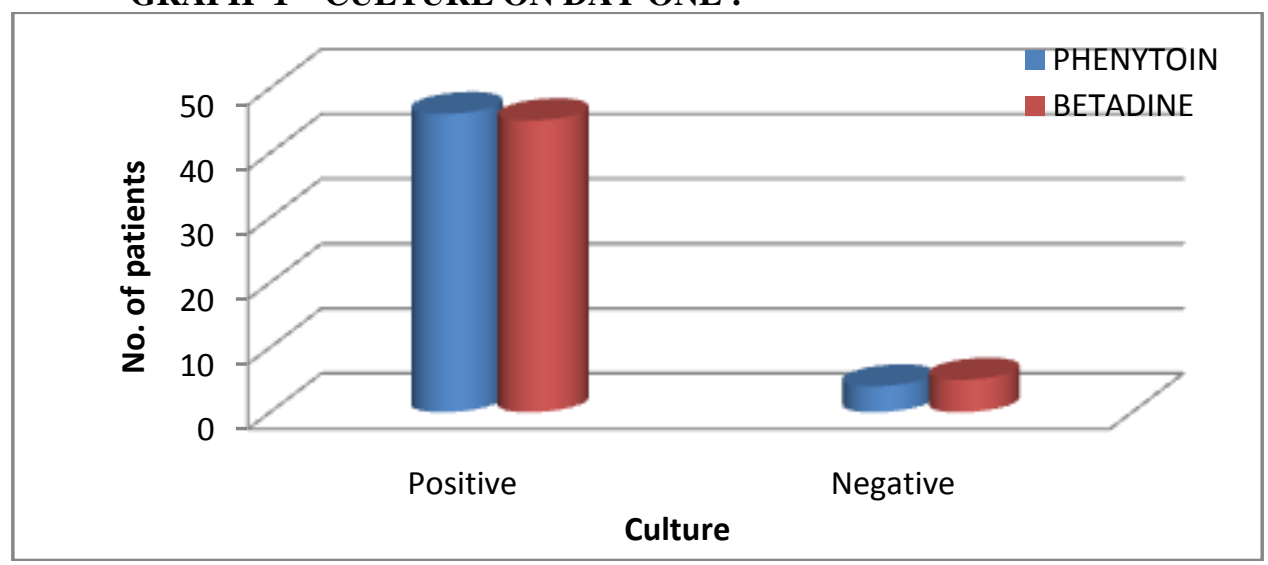

In our study on day one, $46(92 \%)$ patients in study group showed growth on culture and $4(8 \%)$ patients showed no bacterial growth. In control group $45(90 \%)$ patients showed growth on culture media and remaining $5(10 \%)$ showed no g rowth.There was no significant difference between two groups in positive culture growth on day one $(\mathrm{P}-0.727)$

Table 2 : Culture on Day 14

\begin{tabular}{|c|c|c|c|c|c|}
\hline & & & \multicolumn{2}{|l|}{ DRESSING } & \multirow[t]{2}{*}{ Total } \\
\hline & & & PHENYTOIN & $\begin{array}{l}\text { BETADIN } \\
\text { E }\end{array}$ & \\
\hline \multirow[t]{4}{*}{ D14_CUL } & \multirow[t]{2}{*}{$+\mathrm{Ve}$} & Count & 25 & 45 & 70 \\
\hline & & $\begin{array}{ll}\% & \text { within } \\
\text { DRESSING } & \end{array}$ & $50.0 \%$ & $90.0 \%$ & $70.0 \%$ \\
\hline & \multirow[t]{2}{*}{$-\mathrm{Ve}$} & Count & 25 & 5 & 30 \\
\hline & & $\begin{array}{ll}\% & \text { within } \\
\text { DRESSING } & \end{array}$ & $50.0 \%$ & $10.0 \%$ & $30.0 \%$ \\
\hline \multirow{2}{*}{\multicolumn{2}{|c|}{ Total }} & Count & 50 & 50 & 100 \\
\hline & & $\begin{array}{ll}\% & \text { within } \\
\text { DRESSING } & \end{array}$ & $100.0 \%$ & $100.0 \%$ & $100.0 \%$ \\
\hline
\end{tabular}

Cramer's V Value - $0.436 P-0.000$

Graph 2 - Culture Conversion On Day 14 :

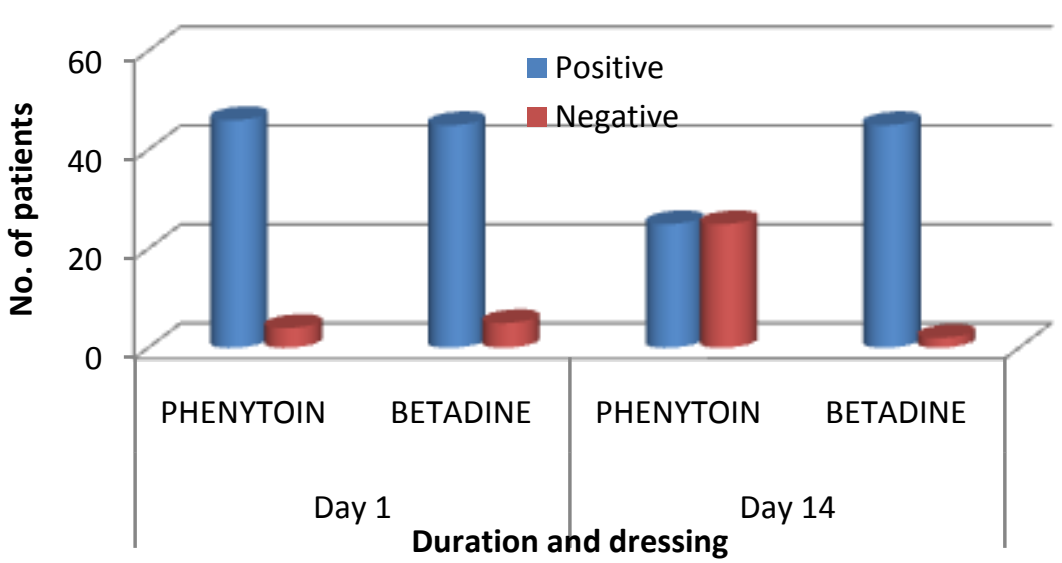


In our study, culture conversion from positive to negative is statistically significant $(\mathrm{p}<0.05)$ in phenytoin group when compared to povidone iodine group. In study group , 45 patients out of 50 are culture positive on day one are reduced to 25 patients ( $92 \%$ to $50 \%$ ) on day 14 and there is no conversion of bacterial growth is noted in control group..

\begin{tabular}{|l|l|l|l|l|l|}
\hline \multicolumn{7}{|c|}{ Table 3 : Wound area on Day one and Day 14 $\left.\mathbf{~ ( ~ m m ~}^{\mathbf{2}}\right)$} \\
\hline & DRESSING & $\mathrm{N}$ & Mean & Std. Deviation & Std. Error Mean \\
\hline \multirow{2}{*}{ D1_AREA } & PHENYTOIN & 50 & 13621.3200 & 24288.57612 & 3434.92338 \\
\cline { 2 - 7 } & BETADINE & 50 & 11410.4460 & 14808.12634 & 2094.18531 \\
\hline \multirow{2}{*}{ D14_AREA } & PHENYTOIN & 50 & 6645.1820 & 14655.56203 & 2072.60946 \\
\cline { 2 - 6 } & BETADINE & 50 & 9449.7044 & 13176.93359 & 1863.49982 \\
\hline
\end{tabular}

Graph 3 : Course Of Reduction In Wound Area

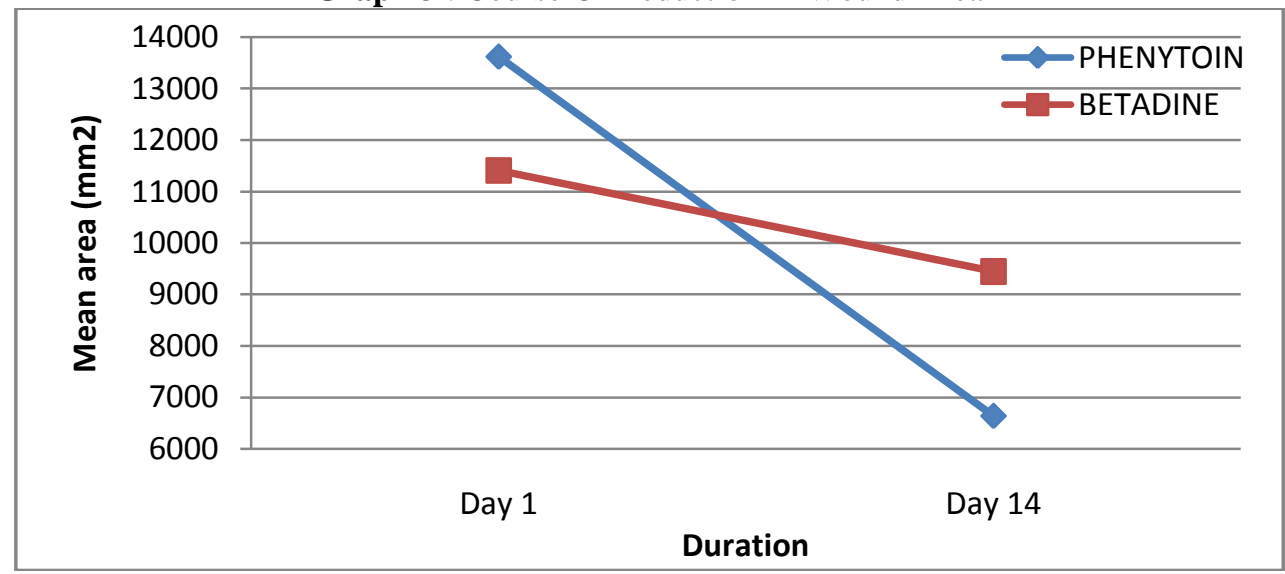

In our study, there is no statistically significant difference ( $\mathrm{P}-0.584$ ) between the study (13621.32 $\mathrm{mm}^{2}$ ) and control $\left(11410.44 \mathrm{~mm}^{2}\right)$ in the initial wound area (day 1$)$ and on day $14(\mathrm{P}-0.317)$ on wound area which is in study group ( $6645 \mathrm{~mm}^{2}$ ) when compared to control group $\left(9449.70 \mathrm{~mm}^{2}\right)$ but there is very significant faster reduction in size in phenytoin group compared to povidone iodine group

Table 4 - Mean reduction in wound area $\left(\mathrm{mm}^{2}\right)$

\begin{tabular}{|l|l|l|l|l|l|}
\hline & DRESSING & $\mathrm{N}$ & Mean & Std. Deviation & Std. Error Mean \\
\hline \multirow{2}{*}{ REDUCTION_SIZE } & PHENYTOIN & 50 & $\begin{array}{l}6976.138 \\
0\end{array}$ & 10193.51978 & 1441.58139 \\
& & & & \\
\cline { 2 - 7 } & BETADINE & 50 & $\begin{array}{l}1960.741 \\
6\end{array}$ & 1982.96866 & 280.43412 \\
\hline $\begin{array}{l}\text { REDUCTION IN } \\
\text { PERCENTAGE }\end{array}$ & PHENYTOIN & 50 & 62.1311 & 12.03716 & 1.70231 \\
\cline { 2 - 7 } & BETADINE & 50 & 18.5397 & 7.99421 & 1.13055 \\
\hline
\end{tabular}

Graph 4 : Mean Reduction In Wound Between Two Groups

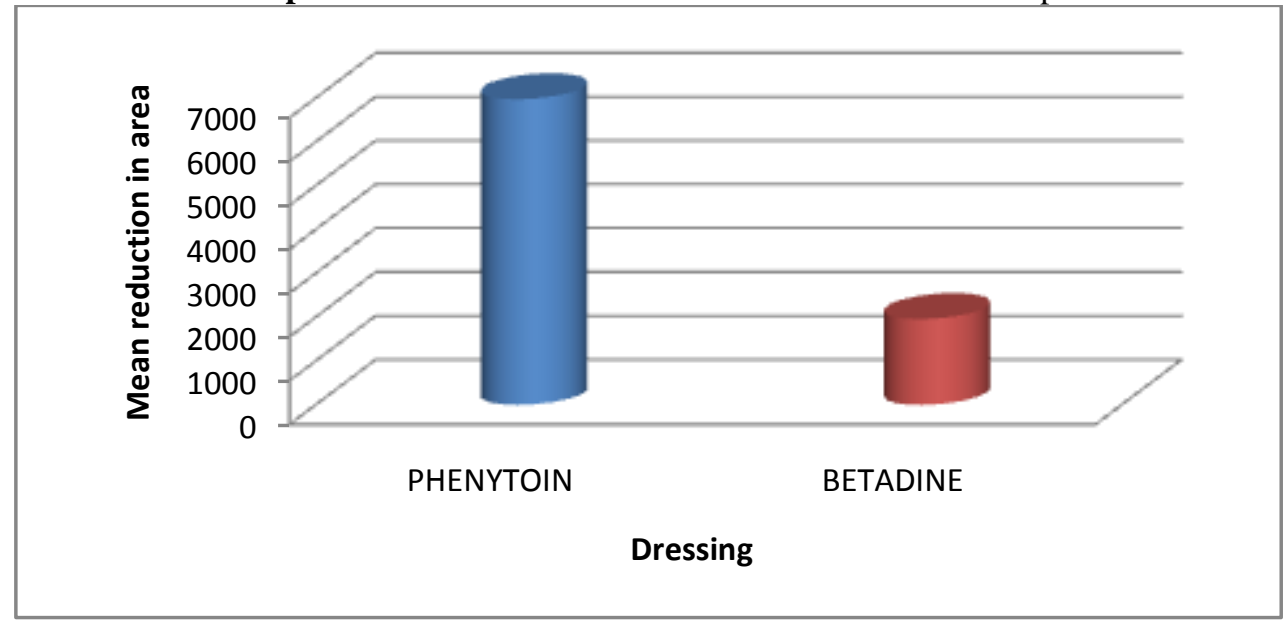




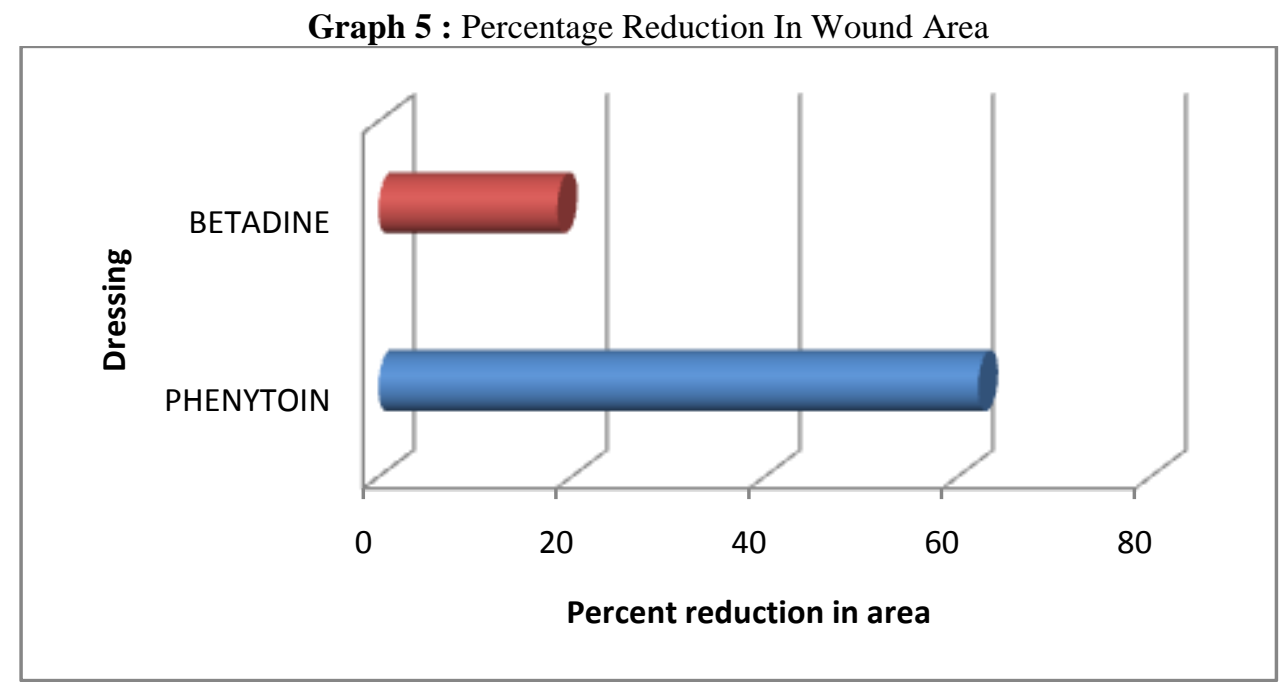

In our study, we have observed that mean reduction in ulcer size in phenytoin $\left(6976.13 \pm 1441.5 \mathrm{~mm}^{2}\right)$ is statistically significant compared to povidone iodine group $\left(1960 \pm 280.4 \mathrm{~mm}^{2}\right)$ and there is $62.1 \pm 1.7 \%$ reduction in size in phenytoin group compared to $18.5 \pm 1.1 \%$ which is statistically significant

Figure 1 : ulcer 1 on day one
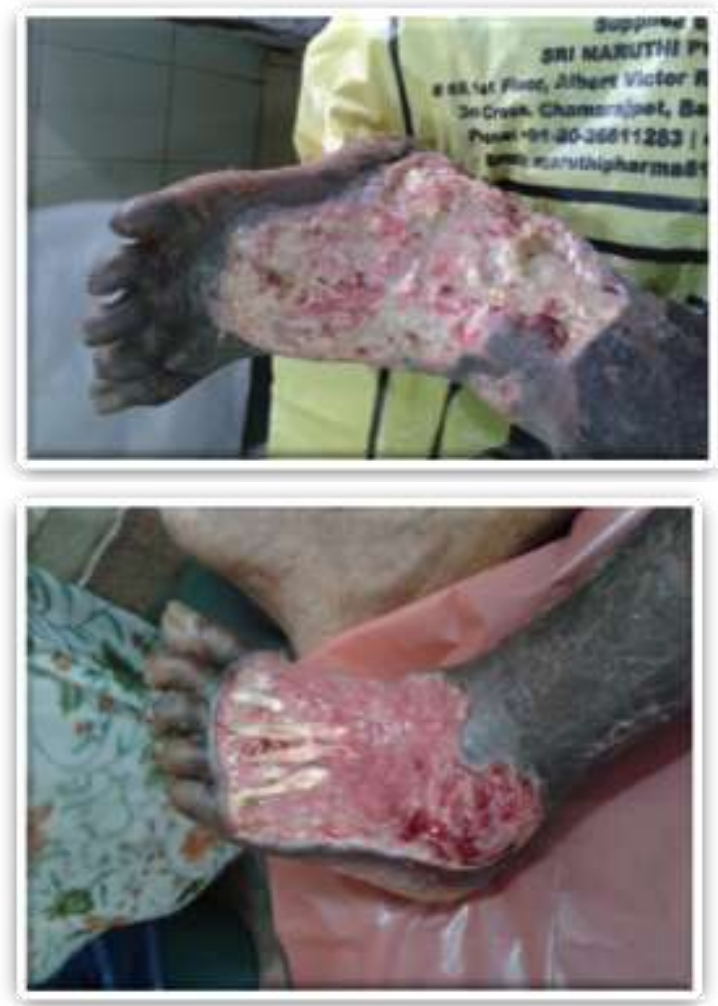

Figure 3 : Ulcer 2 on day 1
Figure 2 : ulcer 1 on day 14
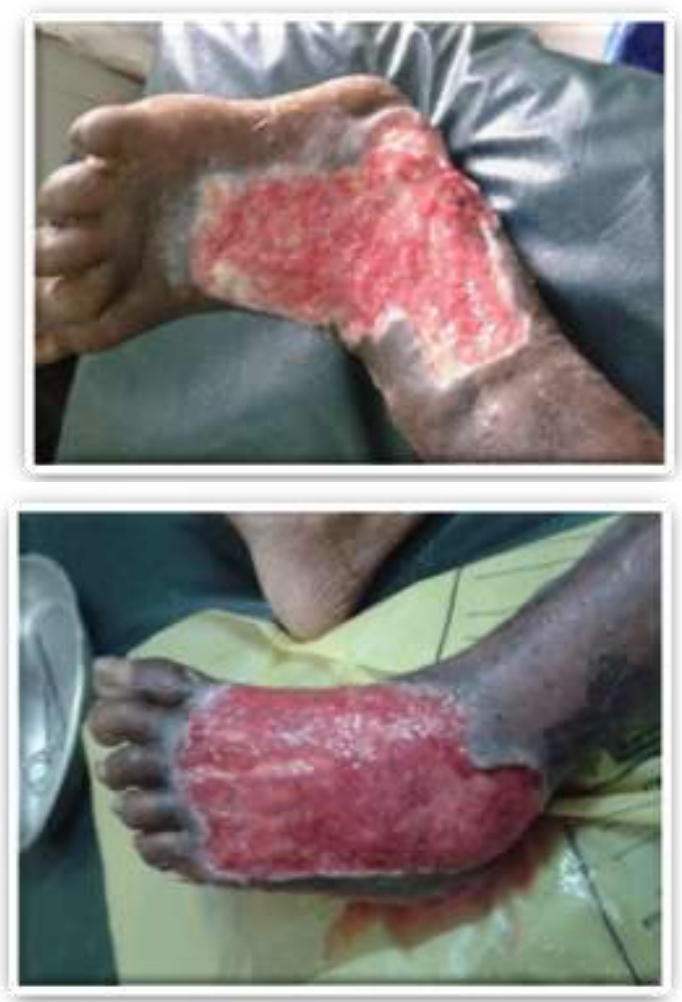

Figure 4 : Ulcer 2 on day 14

\section{Phenytoin :}

In 1938, Meritt and Putnam published their note worthy. Data using phenytoin to treat major, absence and psychic equivalent seizures. Since that time phenytoin has been considered highly effective anticonvulsant . Even decades later it continues to be a highly effective anticonvulsant and anti arrhythmic (10). Phenytoin is type 1B anti arrhythmic and also used in treatment of trigeminal neuralgia Phenytoin has been used to treat ulcers epidermolysis bullosa and inflammatory condition. Numerous allergy and proliferate, idiosyncratic cutaneous side effects have been reported with its use (11). A frequent observed and unwanted side effect of phenytoin, is gingival hyperplasia, specially in children( 12) 
This side effect suggested that phenytoin can induce the growth of connective tissue, and may have the ability to promote wound healing. The beneficial effect of phenytoin. In wound healing had been reported in 1945 and was observed in the first clinical trial for gingival wounds in 1958. Since then, the effectiveness of topical phenytoin has been confirmed by several clinical trials for different types of wounds.

\title{
Postulated Mechanisms of Action:
}

Wound healing is a complicated process requiring the collaboration of many groups of cells and the mechanism by which phenytoin promotes wound healing is not fully understood, but several theories have been proposed mechanisms may include $(13,14)$

- Stimulation of fibroblast proliferation.

- Enhancing the formation of granulation tissue.

- Decreasing collagenase activity,

- Inhibition of glucocorticoid activity

- Direct or indirect antibacterial activity by affecting inflammatory cells

- Phenytoin increases gene expression of the platelet derived growth factor $\mathrm{p}$ chain in macrophage and monocytes.

Clinical Experience

\section{War and Non-War Wounds}

One open trial evaluated 19 patients with missile wounds and 6 patients with refractory ulcers.(12) All wounds were debrided if needed and the wounds were cleansed with normal saline, dried, and covered with a thin layer of phenytoin sodium powder on a daily basis and the course of treatment ranged from 2 to 4 weeks. 22 of 25 patients had complete wound healing. Mean healing time for the missile wounds was 2 weeks, compared to 6-8 weeks for historical controls. The healing time for patients with refractory wounds was 4 weeks.

\section{Burns}

In a study reported by Lodha ,two burn surfaces were evaluated for each of 50 patients with second-and third-degree burns. One burn surface on each patient was dressed with either $1 \%$ silver sulfadiazine cream or with a thin layer of phenytoin powder. At 20 days, the burn area was reduced by $89.6 \%$ for the phenytoin group compared to $56.9 \%$ for the silver sulfadiazine group $(\mathrm{p}<0.001)$. Grafting was $100 \%$ successful for phenytoin treated sites versus $70 \%$ of the sites treated with silver sulfadiazine.

Second and third degree burn wounds were also treated with phenytoin and compared to silver sulfadiazine cream. In second degree burns, the percentage of reduction in mean burn area was $89.6 \%$ for phenytoin sites versus $56.9 \%$ for the silver sulfadiazine treated wounds $(p=<0.001)(15,16)$

\begin{abstract}
Abscess Cavities
Forty patients with gluteal abscess secondary to intramuscular injections were studied by Lodha et al (17). Twenty patients were assigned to receive topical phenytoin and a dry dressing. The 20 control cases were first treated with $15 \%$ Eusol chlorinated lime and boric acid). The cavities were then washed with $4 \%$ urea and dressed with normal saline. The mean rate of reduction of wound area was significantly better for the phenytoin group compared to the control group on day $10(\mathrm{p}<0.05 \%)$ and day $20(\mathrm{p}<0.01 \%)$. The mean volume reduction rates were also better for phenytoin group compared to control group on day 10 and day 20 $(\mathrm{p}<0.005 \%)$. By day 20, 17 patients of the phenytoin group had treated completely, compared with only 1 in the control group.
\end{abstract}

\section{Diabetic Foot Ulcers}

A prospective, controlled trial examined the use of topical phenytoin versus control therapy in 100 noninsulin dependent diabetic patients with foot ulcers (18). In the control group $(n=50)$, a sterile occlusive dressing was applied daily. In the phenytoin group $(n=50)$, phenytoin powder was applied in a "thin layer" to the ulcer surface, then dry dressing daily. Mean healing time was 21 days in the phenytoin group compared to 45 days in the control group $(\mathrm{p}<0.05 \%)$. Topical Phenytoin in diabetic foot ulcer, a study conducted in 1991 by Muthukumarswamy MG et- al, showed the mean time of complete healing of diabetic foot ulcers were 21days with Phenytoin dressing and 45 days with sterile occlusive dressing. The difference seen were statistically significant $(\mathrm{P}<0.05)(4)$.

Topical Phenytoin in Diabetic ulcer, a double blind control study conducted by Pai MRSM, Shrivastava N, Kotian MS , showed that there was an acceleration of wound healing in the Phenytoin group particularly around 3rd week but overall reduction in size of the ulcer was not statistically significant $(\mathrm{P}>0.05)(5)$ 


\section{Trophic Leprosy Ulcers}

Bansal and Mukul (9) compared the wound healing effects of topical phenytoin with normal saline in 100 patients with 110 ulcers. Fifty patients were assigned to the topical phenytoin group and 50 to saline group over a 4 week study period. After 4 weeks, the mean reduction in ulcer volume in the phenytoin group was 72.1 $\%$ compared to $55.5 \%$ in the control group $(\mathrm{p}<0.001)$. The other study by Menezes et al (19) involved a total of 30 patients. Fourteen patients were assigned to topical phenytoin with immobilization and 16 patients to immobilization alone. Both groups showed a significant reduction in depth and surface dimensions of the ulcers at the end of a 3 week study period $(\mathrm{p}<0.01)$. The decrease in ulcer depth in the phenytoin group was not statistically significant compared to the control group. However, the rate of decrease in surface dimensions was significant $(\mathrm{p}<0.05)$ in the phenytoin group compared to the controls.

\section{Chronic Skin Ulcers}

Pendse et al (8) studied a total of 75 patients with various types of wounds including burn, post cellulitic, traumatic, amputation stump, postoperative and nonspecific etiology. Forty patients were included in the phenytoin group and 35 patients in the saline dressing group. Fifty percent of the phenytoin-treated wounds showed negative cultures by day 7 vs $17 \%$ of the saline-treated group. By the end of the 4-week treatment period, 29 of 40 phenytoin-treated ulcers had healed compared to 10 of 35 controls.

\section{Stage II Decubitus Ulcers in the Elderly}

A recent study by Rhodes et al (20) evaluated the wound healing effects of topical phenytoin in comparison to collagen (DuoDerm), or triple antibiotic ointment (TAO). Forty-seven nursing home patients were included in the study. Both the phenytoin and standard treatment groups showed progress in wound healing but the phenytoin group healed faster. In the phenytoin group, healthy granulation tissue appeared within 2 to 7 days (compared to 6 to 21 days for the other two groups). The average time of healing was significantly shorter for the phenytoin group compared to the standard groups $(p<0.005)$. The authors of this study concluded that the use of topical phenytoin can accelerate the healing of pressure sores, and more importantly, can prevent stage II ulcers from progressing to stage III or IV.

\section{Topical Phenytoin Preparation Reported side effect}

Topical phenytoin used in wound therapy appeared to be well tolerated. Its adverse effects were used in clinical trials. systemic absorption was considered insignificant. Allergic reaction to topical phenytoin is rare. Formation of thin layer of phenytoin powder on the ulcer, initial burning sensation, skin rash are the other side effects of topical phenytoin which are resolved when application stopped $(12,15,20)$ No adverse reaction reported $(20,21,22)$.

\section{Discussion}

It is every surgeon's desire that after dressing the wound, it should heal without any complications. Successful wound dressing should keep the wound moist and be devoid of any adverse reactions such as infection, maceration and allergy. Diabetic foot ulcers are stuck in inflammation phase and shows cessation of epidermal growth or migration over the wound surface.

Phenytoin dressing has shown great promise as a procedure for healing of chronic wounds (Venous ulcers, pressure sores, superficial burn wounds, small donor site wounds and minor abrasions). Phenytoin acts by stimulating fibroblasts enhancing granulation tissue formation, decreasing collagenase activity (6)

In the present study, an attempt has been made to establish better healing rates with use of phenytoin dressing in diabetic foot ulcer. In this study the base line characteristics such as age, sex and location of the ulcer were similar in the patients who received phenytoin dressing in the study group and in patients who received povidone iodine dressing in the control group.

This study is a comparative study which is aimed to document the safety and performance of phenytoin dressing in the treatment of established diabetic foot ulcers. The non healing ulcers should be of more than 4 weeks duration and less than $5 \%$ of body surface area. The treatment period was 2 weeks. The mean wound area reduced from $13621.3 \pm 1441.5 \mathrm{~mm}^{2}$ to $6645.1 \pm 280.4 \mathrm{~mm}^{2}$ in patients dressed with topical phenytoin. Percentage of reduction in wound size is $62.1 \pm 1.7 \%$ compared to $18.5 \pm 1.1 \%$ reduction in control group. This study demonstrates that treatment of diabetic foot ulcer with topical phenytoin dressing results in considerable and rapid wound area reduction and reduces infection rate in ulcer.

However, the final area of the ulcer (in $\mathrm{mm}^{2}$ ) was not reduced in patients with phenytoin dressing group as compared to the patients in povidone iodine group but the percentage reduction in the area of the ulcer was more in the phenytoin dressing group $62.1 \pm 1.7 \%$ as compared to the control group $18.5 \pm 1.1 \%$ and this difference was statistically significant $(\mathrm{p}=<0.001)$. 
The following formula was applied to calculate $\%$ reduction in area of wound after 2 weeks period in both cases and controls.

$\%$ Reduction of wound after 2 weeks $=\quad \begin{gathered}\text { Initial area }- \text { Final area } \\ \text { - }\end{gathered}$

In our study it was noticed that conversion from positive culture to negative culture growth on day 14 was significant in patients dressed with topical phenytoin $(\mathrm{p}=0.00)$. El Zayat $(21)$ in his study reported reduction of wound contamination with topical phenytoin and postulated it is not a direct anti bacterial effect, but rather a change in the $\mathrm{pH}$ and improvement in local circulation. However Lodha et al (16) suggested that phenytoin may have a direct antibacterial effect. Further in vivo and in vitro studies are required to establish the anti-infective effect of topical phenytoin dressing and its mechanism of action.

In this study it was noticed that compared to povidone iodine, topical phenytoin is more effective in inhibiting wound infection. This conclusion was based on the following findings: earlier appearance of granulation tissue, earlier disappearance of wound discharge and post treatment wound cultures were negative in 25 of 50 patients who were treated with topical phenytoin, but there is no conversion noted in patients wound cultures in control group, who received povidone iodine dressing

Systemic absorption of phenytoin on topical use in diabetic ulcer is not significant. Most studies that have monitored serum phenytoin levels during topical application have shown the levels to be undetectable (20) Other known side effects of use of topical phenytoin in diabetic ulcer are transient burning sensation initially and hypergranulation. There were no side effects noted in the patients dressed with topical phenytoin in our study.

Overall this study shows that phenytoin dressing is safe and effective in treating chronic foot ulcers. This study was conducted only for 2 weeks and complete epithelialization and wound reduction was not awaited for.

\section{Limitations of our study}

- Not a blinded study

- Follow up is short to derive conclusion on long term healing of the ulcers.

\section{Scope for further study}

There is further scope of study among infective diabetic wound with respect to anti-infective properties of topical phenytoin dressing

\section{Conclusion}

With the use of topical phenytoin dressing in comparison with the povidone iodine dressing for the treatment of diabetic foot ulcers, the following conclusions were derived;

- Topical phenytoin dressing showed faster and better healing rates among the study group.

- Area reduction and percentage reduction was better in topical phenytoin dressing group.

- There was no adverse effect or reactions seen when topical phenytoin dressing was applied over the ulcer.

- Appearance of granulation tissue was earlier as compared to $5 \%$ povidone Iodine dressing

- Topical phenytoin dressing may have anti infective effect.

\section{Bibilography}

1. Boulton AJM. The diabetic foot. Medical clinic of north America no. 1988; 72 (6): 1513 - 30

2. Most RS, Sinnock P, Epidemiology of lower extremity amputation in diabetic individuals. Diabetes Care 1983; $2: 87-91$.

3. Mann CV, Russel RCG, Williams NS. Bailey and Love's Short Practice of Surgery. 24th Ed. New York: Hodder Arnold Publication; 1995.

4. Muthukumarswamy MG, Shivakumar G, Manoharan G. Topical phenytoin in diabetic foot ulcer. Diabetic care. October 1991; 14(10): 909 11

5. Pai MRSM, Shrivastava N, Kotian MS. Topical Phenytoin in diabetic ulcer. Double blind control trial. Ind J Med Nov 2001; 55(11): 513-99.

6. Bhatia A, Surya P. Topical Phenytoin in wound healing. Dermatology online Journal. 10(1) : 5 .

7. Shaw I. Hughes CM. Lagan KM, Bell P M. The clinical effect of tropical phenytoin on wound healing : a systemic review. B. J of Dermatology 2007: 157: $997-04$.

8. Pendse A K, et al. Topical pheytoin in wound healing. International Journal of Dermatology 1993: $32(3): 214-19$.

9. Bansal and Mukul. Phenytoin treatment of ulcer. International Journal of Dermatology. 1993; 32(3): 210-13.American diabetes association: Clinical practice recommendations 2002. Diabetes Care 2004; 27: 51

10. R.S. Satoskar, S.D Bhandarker, S.S Ainapure, Textbook of Pharmocology And pharmacotherapeutics chapter 7 Drug effective in convulsive disorders $17^{\text {th }}$ Editn Page No. 118-134

11. Noah Scheinfeld MD Phenytoin in cutaneous medicine : its uses, mechanism and side effects DOJ 9(3):6

12. Modagheghs, Salchian B, Tavassoli M, et al use of phenytoin in healing of war and non war wounds Int. J. Dermatol 1980; 28(5): 34750 . 
13. Talas G, Brown RA, McGrouther A. Role of phenytoin in wound healing-a wound pharmacology perspective. Biochem Pharmacol

14. Genever PG, Cunliffe WJ, Wood EJ. Influence of the extracellular matrix on fibroblast responsiveness to phenytoin using In vitro wound healing models. Br J of Dermatol 1996; 133:231-35

15. Lodha SC, New application of an old drug: topical phenytoin for burns, J. Burns care Rehabil 1991; 12 (1): 96.

16. Mendiola-Gonzalez JF, Espejo-Plascencia I, Chapa-Alvarez JR, Rodriguez- Noriega E. Sodium diphenylhydantoin in burns effect on pain and healing. Invest Med Int 1983;10:449-51

17. Lodha SC, Lohiya ML, Vyas MC, et al. Role of phenytoin in healing of large abscess cavities. Br. J. Surg. 1991; 78: 105-108.

18. James WB. The Diabetic Foot. In Surgery of the foot and ankle (Mann RA, Couglin MJ.) :877-953.

19. Menezes J., Rajendran A, Jacob A.J and Vaz. M. The use of topical phenytoin as an adjuant in the treatment of trophic ulcers, South east Asia J. Trap. Med public health 1993; 24(2): 340-42.

20. Rhodes RS, Heyneman CA, Culbersten VL, Wilson SE, Phatak HM, Topical phenytoin treatment of stage II decubitus ulcers in the elderly, Ann Pharmaco therapy 2001; 35: 675-81.

21. Ashima Bhatia MD, Surya Prakash DVD, topical phenytoin for wound healing , dermatology online journal 2009;10(1):5

22. El Zayat S. preliminary experience with topical phenytoin in wound healing in war zone.Milit Med 1989;154:178-180 